\title{
Minimal fat renal angiomyolipoma with central scar and stellate calcification mimicking a calyceal calculus
}

Eugene Low $\underline{L}^{1}$, MBBS, FRCR, Cher Heng $\underline{\operatorname{Tan}}^{1}$, MBBS, FRCR, Bernard $\underline{H o}^{2}$, MBBS, FRCPath, Simon Chong $^{3}$, MB BCh BAO, MRCS

ABSTRACT Renal angiomyolipomas are benign neoplasms composed of varying amounts of adipose tissue, smooth muscles and blood vessels. They typically contain macroscopic fat, which is seen as negative attenuation on computed tomography. Calcification and scarring is rarely seen in renal angiomyolipomas. We report the case of a 40-year-old man who was found to have a renal angiomyolipoma with a central stellate scar and focal calcification. The lesion was initially misdiagnosed as a calyceal calculus.

Keywords: calcification, renal tumour, scar

\section{INTRODUCTION}

Renal angiomyolipomas (AMLs), the most common mesenchymal renal tumours, are typically composed of varying amounts of mature adipose tissue, smooth muscle and blood vessels. ${ }^{(1)}$ The incidence of AMLs has been reported to be $0.1 \%$ in men and $0.2 \%$ in women in the general population without tuberous sclerosis.(2) AMLs that occur in association with tuberous sclerosis and renal involvement is found in most patients approximately $69 \%-80 \%$ of patients with tuberous sclerosis have AMLs. ${ }^{(3)}$ AMLs usually contain macroscopic fat, although in rare cases, minimal fat has been encountered on computed tomography $(\mathrm{CT}) .{ }^{(4)}$ Although calcification and scarring are very rare features of AMLs, some studies have reported calcifications in patients with renal AMLs; however, these calcifications did not appear to be large and coarse enough to mimic a renal calculus. ${ }^{(5)}$ Herein, we report the case of a patient with renal $A M L$ that had stellate calcification within a central scar seen on $\mathrm{CT}$, which mimicked a large calyceal calculus on plain radiographs.

\section{CASE REPORT}

A 40-year-old man with no significant past history presented with an initial symptom of right back pain at the lumbar region. Radiography revealed a $1.7-\mathrm{cm}$ calcification in the right lumbar region (Fig. 1). The patient subsequently underwent intravenous urography (IVU) and was diagnosed with a right upper pole calyceal calculus, although no significant hydronephrosis was noted (Fig. 2). He then underwent two successive courses of extracorporeal shock wave lithotripsy (ESWL).

As there was neither radiographic nor clinical response to ESWL, CT urography was performed approximately three months after the initial ESWL. CT urography demonstrated a well-circumscribed, lobulated, enhancing mass measuring $2.9 \mathrm{~cm} \times 4.4 \mathrm{~cm}$ in size, located within the parenchyma of

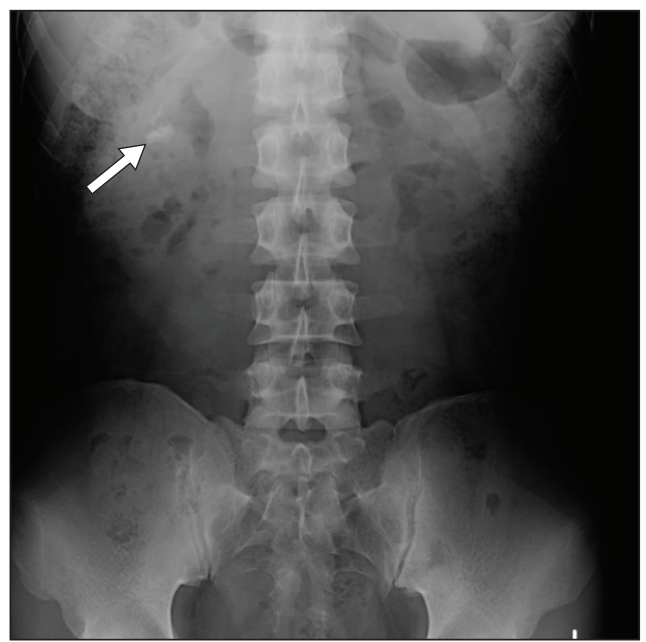

Fig. 1 Plain frontal supine radiograph of the abdomen shows irregular coarse calcification (arrow) projected over the right renal shadow, which was deemed to represent a calyceal calculus.

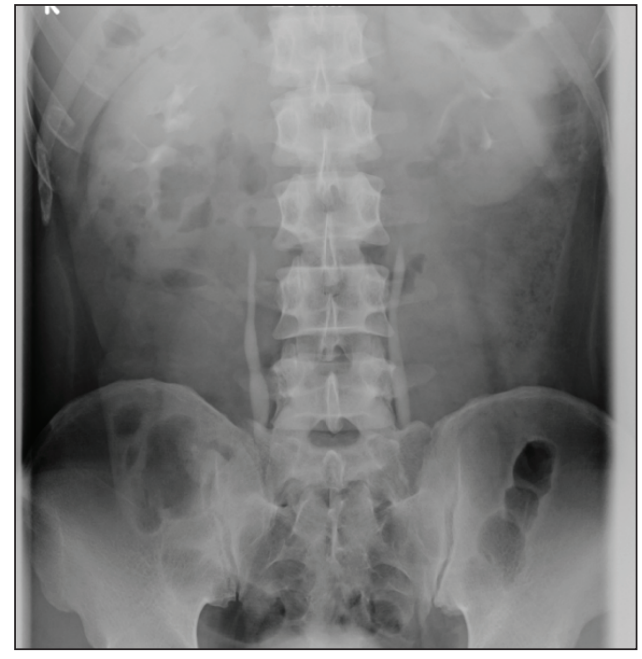

Fig. 2 Excretory phase radiograph taken during intravenous urography shows no significant hydronephrosis. The coarse calcification, superimposed on the opacified calyceal system, was deemed to confirm the presence of a calyceal calculus.

${ }^{1}$ Department of Diagnostic Radiology, ${ }^{2}$ Department of Pathology, ${ }^{3}$ Department of Urology, Tan Tock Seng Hospital, Singapore

Correspondence: Dr Eugene Low, Registrar, Department of Diagnostic Radiology, Tan Tock Seng Hospital, 11 Jalan Tan Tock Seng, Singapore 308133. low_eugene@hotmail.com 

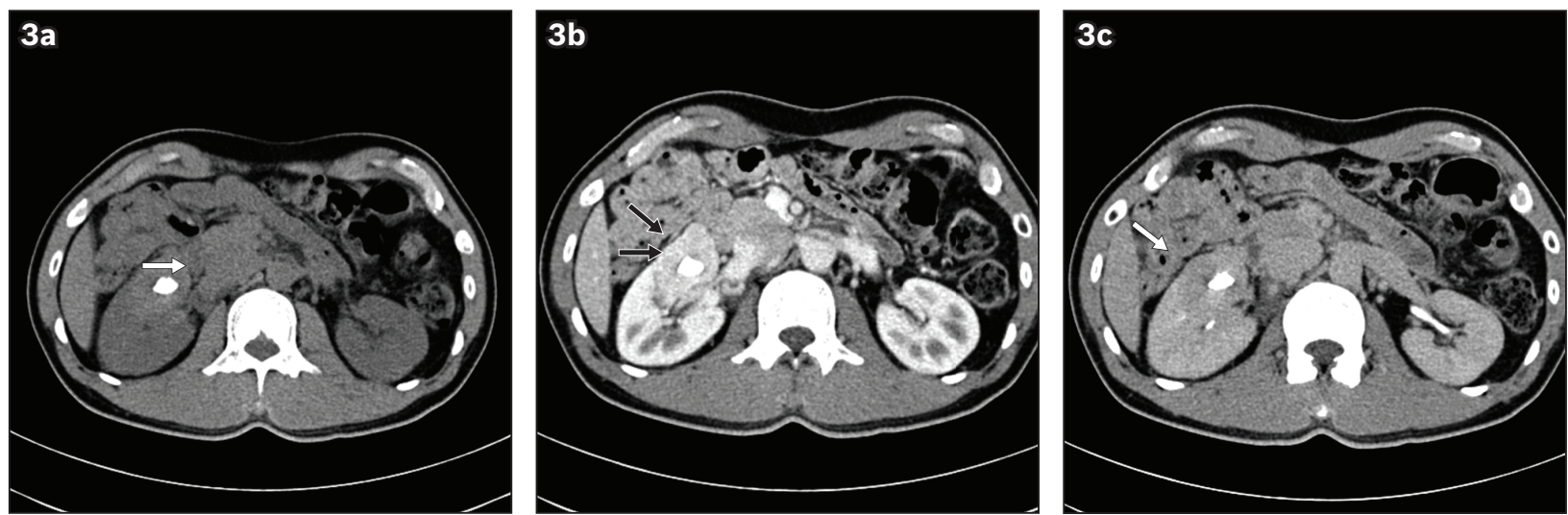

Fig. 3 CT urography performed after two successive treatments with extracorporeal shock wave lithotripsy. (a) Axial precontrast CT image shows focal coarse calcification (arrow) within the anterior cortex of the right kidney. A thin rim of hypoattenuation observed adjacent to the calcification could have represented either scarring or beam-hardening artefact. (b) Axial postcontrast CT image shows a heterogeneously enhancing, lobulated, soft-tissue mass (arrows), with coarse calcification and a hypoattenuating rim surrounding it. (c) Axial delayed CT image shows washout of contrast from the soft-tissue mass (arrow).
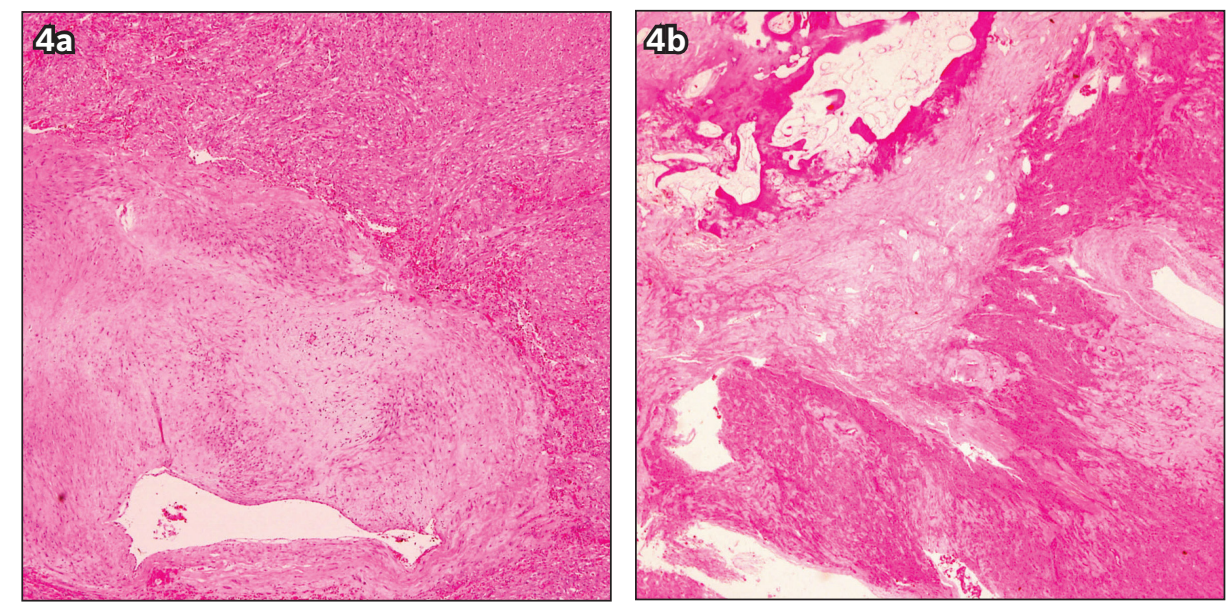

Fig. 4 Photomicrographs of the resected lesion show (a) a prominently smooth muscle component and abnormally large, thick-walled vessels with eccentrically placed lumens (Haematoxylin \& eosin, $\times 40$ ); and (b) a central stellate area of fibrous degeneration and ossification (Haematoxylin \& eosin, $\times 20$ ).

the right kidney (Fig. 3). The mass contained a central stellate scar with calcification, which accounted for the presumed calyceal calculus observed on plain radiography and IVU. Oncocytoma and renal cell carcinoma (RCC) were considered among the differential diagnoses.

The patient opted for surgical removal of the mass instead of a biopsy. Intraoperatively, a large tumour measuring approximately $6 \mathrm{~cm} \times 6 \mathrm{~cm}$ was found in the right upper renal pole. A right total nephrectomy was performed. The mass showed close proximity to the hilar vasculature, and adhesions were encountered around Gerota's fascia. Histolopathological examination of the resected mass revealed spindle cell tumour compatible with a smooth muscle predominant $\mathrm{AML}$ (Fig. 4). Central degenerative changes and focal ossification were observed.

\section{DISCUSSION}

Renal AMLs are typically fat-containing, benign tumours that mostly (95\%) exhibit negative attenuation on CT. ${ }^{(6)}$ On magnetic resonance (MR) imaging, hyperintense adipose tissue on T1-weighted images is a hallmark finding. Areas containing macroscopic fat typically show an 'Indian-ink' artefact along the margins on opposed phase dual-echo images, while on chemical fat-suppressed images, the entire area shows signal loss. However, in cases of minimal fat renal AML, CT and MR imaging features may not differ from those of RCC, since both lesions appear as soft-tissue tumours with contrast enhancement on these modalities. Some authors have, however, found the contrast enhancement patterns of minimal fat renal AML and RCCs to be different, with minimal fat AMLs showing a more homogeneous (79\% of AMLs vs. $5 \%$ of RCCs) and more prolonged (58\% of AMLs vs. $10 \%$ of RCCs) enhancement pattern when compared to RCCs. ${ }^{(7)}$ Still, the enhancement pattern may not exclude hypovascular RCC, particularly if small. ${ }^{(8)}$ There have been previous reports of RCCs containing fat. However, the presence of calcification in our patient made the diagnosis of RCC more likely, since calcification has been reported to occur in $15 \%-20 \%$ of RCCs, and is usually central, amorphous and peripheral, or curvilinear in cystic RCC. ${ }^{(9)}$

The presence of a central stellate scar and focal calcification that resembled a calyceal stone were two key 
misleading features in our patient. A central stellate scar, which is considered a prime feature of oncocytoma, is seen in $30 \%$ of oncocytomas. It typically presents in lesions $>3 \mathrm{~cm}$ in diameter due to the organisation of central infarction and haemorrhage after the tumour growth has outstripped its blood supply. ${ }^{(6)}$ Given that the lesion in our patient had been treated using ESWL prior to being identified as an $A M L$, it is uncertain whether the scar observed during $\mathrm{CT}$ urogram was originally present or had developed as a result of post-treatment changes. There is a possibility that beam-hardening artefacts arising from around the central coarse calcification might have accounted for the thin hypoattenuating rim in our patient, although fibrous degeneration was eventually found on histopathology.

Calcification in AML is rare and has been previously described as peripheral and curvilinear. ${ }^{(10)}$ When present in AMLs, calcifications tend to manifest as small foci. Large, central stellate calcification is unusual for AML and would have been more in keeping with RCC, given that 15\%-20\% of RCCs are known to calcify. ${ }^{(9)}$ As AML is a benign tumour, it does not require routine excision unless symptomatic. Small AMLs $(<4 \mathrm{~cm})$ are typically asymptomatic and generally do not require intervention. It has been suggested that patients with symptomatic AMLs $<4 \mathrm{~cm}$ should undergo digital subtraction angiography and that selective transcatheter renal artery embolisation, enucleation or partial nephrectomy be considered. Asymptomatic AMLs $\geq 4 \mathrm{~cm}$ should be monitored every six months using ultrasonography, and symptomatic lesions $<4 \mathrm{~cm}$ should be monitored till symptoms subside. Should symptoms persist, treatment options would be as mentioned earlier..$^{(1,11)}$

In our patient, given the large size of the lesion and the presence of adhesions around Gerota's fascia, a right total nephrectomy was elected intraoperatively. In retrospect, had the patient preferred otherwise, fine-needle biopsy would have helped avoid radical surgery in view of the radiological findings. Fine-needle biopsy with helical CT guidance has been shown to be accurate for the histopathological evaluation of renal masses, with the associated risk of tumour seeding appearing to be negligible. ${ }^{(12)}$ Fine-needle biopsy has also been recommended for: (a) patients with solid renal tumours that do not have the typical radiological features of RCC; (b) patients with Bosniak category III or IV cystic lesions; (c) patients with locally advanced and metastatic RCC; and (d) patients with tumours that do not require surgery, such as renal metastasis, lymphomas and benign tumours. ${ }^{(13)}$

According to Neuzillet et al, biopsy could be made mandatory for patients with solid renal tumours $<4 \mathrm{~cm}$, as histopathological evaluation, which has a high accuracy rate (92\%), is necessary for tumour management in these patients. ${ }^{(13)}$ However, clinicians need to be aware that cytological diagnosis of AMLs can be difficult, especially when the radiological diagnosis is not clear. ${ }^{(15)}$

To summarise, we herein report the rare case of a patient who had a minimal fat AML with a central stellate scar and coarse calcification. Macroscopic fat is considered a primary feature of renal AMLs. It allows for radiological diagnosis of renal AMLs to be confidently made, in the same way that the presence of calcification within a tumour on imaging studies is thought to be more suggestive of RCC. Our patient was initially misdiagnosed with a calyceal calculus based on radiography and IVU, leading to failed treatment with ESWL. Furthermore, findings on subsequent $\mathrm{CT}$ urograms were indicative of oncocytoma or RCC. Therefore, clinicians should remain alert to the possible variations in the presenting features of renal tumours. Biopsy and histopathological evaluation should be carried out for tumour management of patients with renal masses.

\section{REFERENCES}

1. Oesterling JE, Fishman EK, Goldman SM, Marshall FF. The management of renal angiomyolipoma. J Urol 1986; 135:1121-4.

2. Fujii Y, Ajima J, Oka K, Tosaka A, Takehara Y. Benign renal tumors detected among healthy adults by abdominal ultrasonography. Eur Urol 1995; 27:124-7.

3. Sooriakumaran P, Gibbs P, Coughlin G, et al. Angiomyolipomata: challenges, solutions, and future prospects based on over 100 cases treated. BJU Int 2010; 105:101-6.

4. Hafron J, Fogarty JD, Hoening DM, et al. Imaging characteristics of minimal fat renal angiomyolipoma with histologic correlations. Urology 2005; 66:1155-9.

5. Merran S, Viellefond A, Peyromaure M, Dupuy C. Renal angiomyolipoma with calcification: CT-pathology correlation. Br J Radiol 2004; 77:782-3.

6. Dähnert W. Radiology Review Manual. 6th ed. Philidelphia: Lippincott Williams \& Wilkins, 2007: 940,960-1.

7. Kim JK, Park SY, Shon JH, Cho KS. Angiomyolipoma with minimal fat: differentiation from renal cell carcinoma at biphasic helical CT. Radiology 2004; 230:677-84.

8. Scialpi M, Di Maggio A, Midiri M, et al. Small renal masses: assessment of lesion characterization and vascularity on dynamic contrast-enhanced MR imaging with fat suppression. AJR Am J Roentgenol 2000; 175:751-7.

9. Henderson J, Germany R, Peavy PW, Eastham JA, Venable DD. Fat density in renal cell carcinoma: demonstration with computerized tomography. J Urol 1997; 157:1347-8.

10. Wasserman NF, Ewing SL. Calcified renal oncocytoma. AJR Am J Roentgenol 1983; 141:747-9.

11. Halpenny D, Snow A, McNeill G, Torreggiani WC. The radiological diagnosis and treatment of renal angiomyolipoma-current status. Clin Radiol 2010; 65:99-108.

12. Volpe A, Kachura JR, Geddie WR, et al. Techniques, safety and accuracy of sampling of renal tumors by fine needle aspiration and core biopsy. J Urol 2007; 178:379-86.

13. Lechevallier E, Andre M, Barriol D, et al. Fine-needle percutaneous biopsy of renal masses with helical CT guidance. Radiology 2000; 216:506-10.

14. Neuzillet Y, Lechevallier E, Andre M, Daniel L, Coulange C. Accuracy and clinical role of fine needle percutaneous biopsy with computerized tomography guidance of small (less than $4.0 \mathrm{~cm}$ ) renal masses. J Urol 2004; 171:1802-5.

15. Granter SR, Renshaw AA. Cytological analysis of renal angiomyolipoma: a comparison of radiologically classic and challenging cases. Cancer 1999; $87: 135-40$ 